\title{
A Novel Radiation Enhanced Active Antenna with Switched Dual Circular Polarization
}

\author{
Anne Abeygunasekera ${ }^{\# 1}$, Manju Henry ${ }^{* 2}$, Charles Free ${ }^{\# 3}$ \\ \#Advanced Technology Institute, University of Surrey \\ Guildford, Surrey, GU2 7XH, United Kingdom \\ ${ }^{1} \mathrm{a} \cdot$ abeygunasekera@surrey.ac.uk \\ ${ }^{3} \mathrm{c}$. freedsurrey.ac.uk
}

\begin{abstract}
In this paper we have extended the concept of a travelling wave fed circularly polarized antenna $[1,2]$, to provide an active antenna with the capability to switch between right and left hand polarization. The antenna was fabricated in a multilayer format using a newly available polymer material, with PIN diodes to perform the switching function. Measured data at $15 \mathrm{GHz}$ showed a good return loss in both states, with well behaved radiation characteristics. The design technique offers potential for further development to provide an integrated antenna with high functionality.
\end{abstract}

\section{INTRODUCTION}

The expanding applications for microwave communications have generated a need for compact, high performance antennas that combine the potential for integration with high functionality. The proposed antenna meets these key requirements by utilising a multilayer format that provides a very compact structure, whilst a novel feeding technique that offers opportunities for high functionality. The functionality is achieved by using PIN diodes to switch the direction of the signal in the feed lines. Various possibilities exist for switching between circular and linear polarization, but in this case we have demonstrated the switching between the two hands of circular polarization.

The antenna was fabricated using a new polymer technology to form a multilayer structure. The polymer material had two primary advantages for the present work: (i) the material could be loaded with ceramic to change the dielectric constant; (ii) the material could be laminated, under low pressure and temperature, to form a multilayer circuit. These properties meant that low dielectric constant material could be used beneath the radiation patches, to enhance radiation [2], and a high dielectric material could be used for the feed network to achieve a compact.

\section{ANTENNA STRUCTURE}

The antenna was essentially composed of two dielectric layers, separated by a common ground plane as illustrated in Fig. 1. An overview of the complete antenna, showing the registration of the various layers is given in Fig. 2.The radiating patches were formed on the surface of the layer with low dielectric constant $\left(\varepsilon_{\mathrm{r}}=3\right)$, and the microstrip feed network on the layer with high dielectric constant $\left(\varepsilon_{\mathrm{r}}=6\right)$. Slots were etched in the ground plane prior to lamination. The slot dimensions were designed to provide a characteristic impedance of $50 \Omega$ [3]. The slots were formed into two, parallel annular rings, running beneath the patches. Fig. 3 shows the relative position of the conductors on each layer, and Fig. 6 shows photographs of the top and bottom surfaces of the final structure. Four beam-lead PIN diodes were surface-mounted on the microstrip feed network to route the input signal to one or other of the slotlines, and Fig. 5 shows the surface mounted diodes.

The position of two VIA arrays are shown in Fig. 2. These were used to connect two ends of signal lines on the bottom layer to a short length of line on the top layer, thus enabling signal lines to cross. Note that these VIAs pass through regions of the buried ground plane where the conductor was been removed to prevent the VIAs shorting to ground.

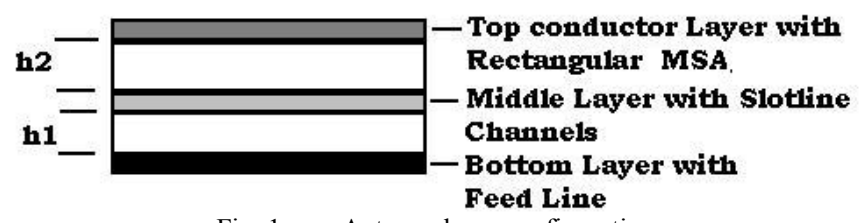

Fig. 1. Antenna layer configuration.

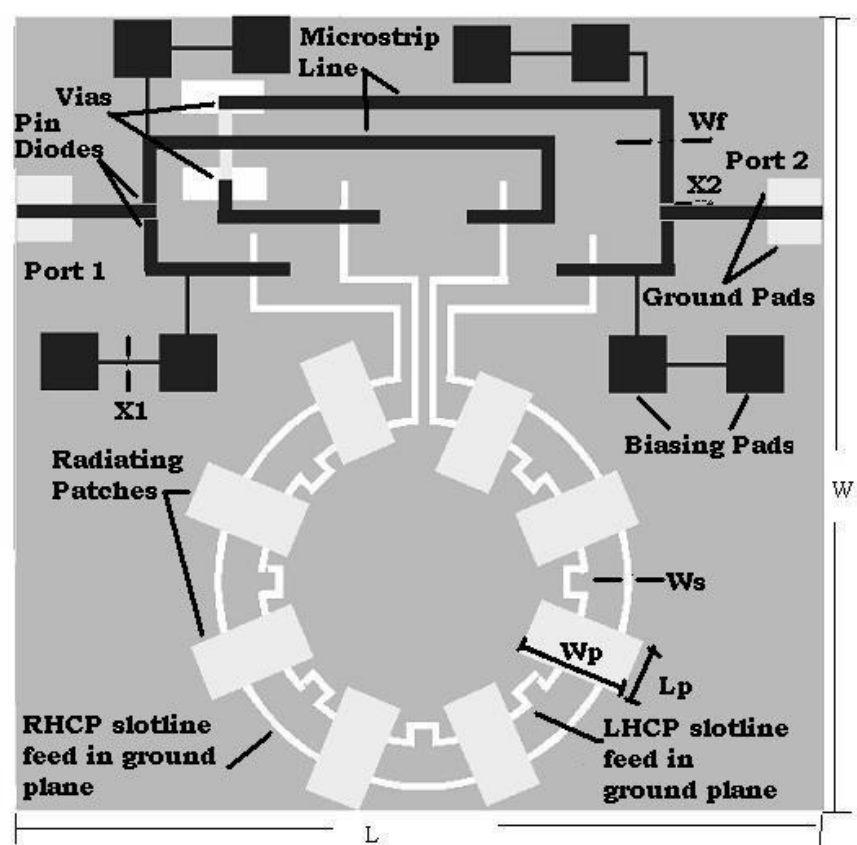

Fig.2. Novel dual CP switched antenna 
Fig 3 shows the position of the slots relative to the patches in more detail.

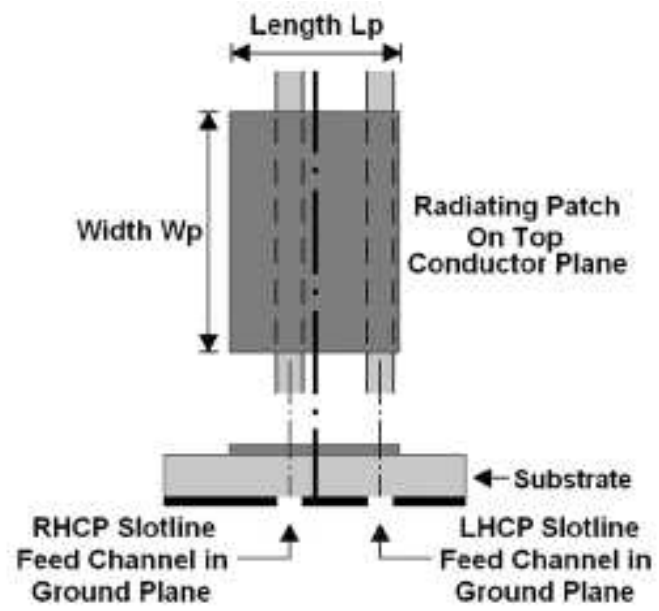

Fig. 3. Dual slotline arrangement[1].

One of the benefits of using the polymer material was that low dielectric material could be selected for the substrate immediately beneath the patches, thus enhancing the radiation.

In order to switch the traveling wave signal between the two rings, and thus change the hand of polarization, PIN diode switching elements were incorporated into the microstrip feed networks as indicated in Fig 4.

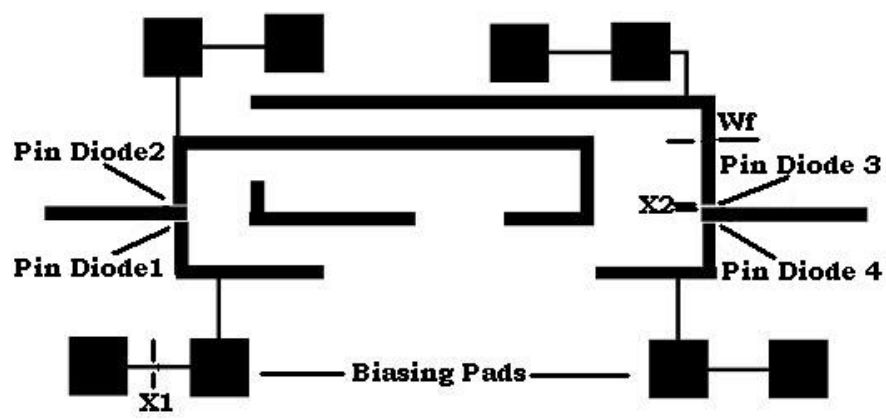

Fig. 4a. Positioning of the Pin Diodes for Switching Polarization

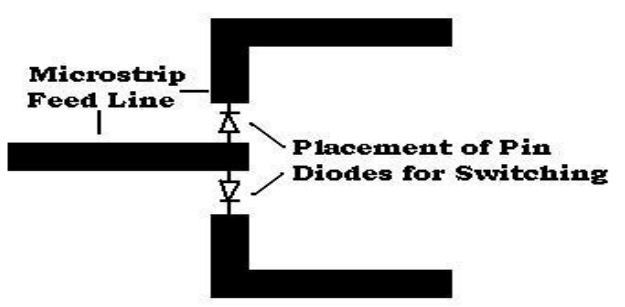

Fig. 4b. Configuration of the pin diodes.

\section{ANTENNA THEORY}

A signal applied to the microstrip line at port 1 of the antenna will be coupled to one of the buried slotline, through microstrip-to-slotline transitions. The states of the PIN diodes will determine which of the slotlines is energized. Energy travelling in a slotline beneath the patches will excite the patches into resonance and cause radiation. Since the annular spacing of the 8 patches is $45^{\circ}$, and the circumference of the ring is $\mathrm{n} \lambda_{\mathrm{g}}$, a circularly polarized signal will be radiated. As the signal travels around the slotline ring, there is successive coupling of energy into the patches. Clearly there will be a progressive loss of energy as the signal propagates around the ring, and to ensure that each patch receives the same level of coupled energy, there is a progressive offset of the patches relative to the slotline, ensuring that the later patches are more strongly coupled to the line. Two slotline channels are provided, so that switching between them enables either righthand, or left-hand, circular polarization to be produced, as indicated in Fig. 1.

\section{IV.FABRICATED ANTENNA}

Photographs of the fabricated antenna are shown in Fig 5.

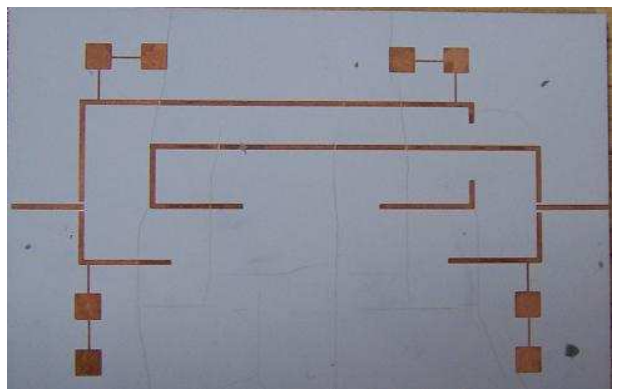

(a)

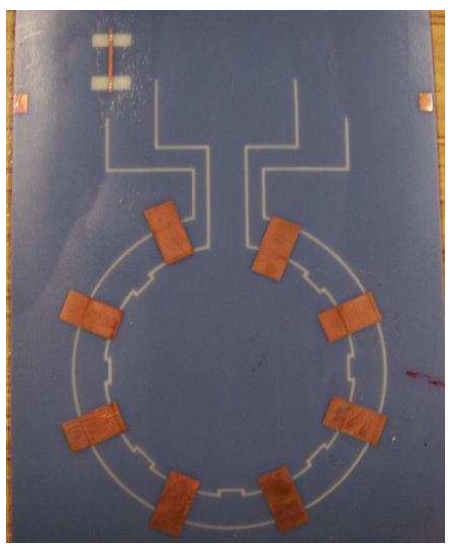

(b)

Fig. 5 Fabricated CP antenna: (a) Bottom view with microstrip feed network; (b) Top view with radiating patches. 
The $15 \mathrm{GHz}$ test antenna was fabricated on polymer material with the following parameters: bottom substrate thickness $\mathrm{h} 1$ $=0.3 \mathrm{~mm}$, substrate dielectric constant $\varepsilon_{\mathrm{r}}=6$; Top substrate thickness $\mathrm{h} 2=2 * 0.127=0.254 \mathrm{~mm}$, substrate dielectric constant $=2.2$. The key antenna dimensions were:

Length of the patch $\mathrm{Wp}=6.74 \mathrm{~mm}$

Width of the patch $\mathrm{Lp}=3.5 \mathrm{~mm}$

Slot width $\mathrm{w}_{\mathrm{s}}=3 \mathrm{~mm}$

$\mathrm{x}_{1}=0.150 \mathrm{~mm}$

$\mathrm{x}_{2}=0.200 \mathrm{~mm}$

Length of the board $\mathrm{L}=50.18 \mathrm{~mm}$

Width of the board $\mathrm{W}=71.933 \mathrm{~mm}$

It should be noted in Fig. 5 that because the polymer dielectric beneath the radiating patches is thin and of low permittivity, the top view also shows the slotline channels in the buried ground plane.

Fig. 6 shows a detailed view of the mounting of the beamlead PIN diodes at one end of the structure.

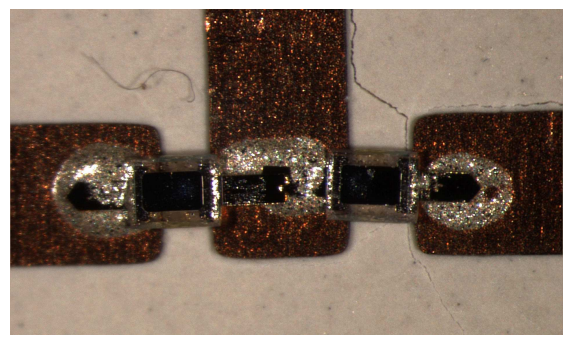

Fig.6. Layout of the pin diodes.

At $15 \mathrm{GHz}$ it was quite important that the diodes were properly positioned across the gaps, so as to minimize stray parasitic effects, and maintain the same transmission characteristics in each state. Otherwise, switching the diodes would cause differences in the transmitted signal levels for the right and left hand polarization.

\section{MEASUREMENT DATA}

The return loss of the antenna for both polarizations is illustrated in Fig. 7. The optimum frequency of operation was $14.95 \mathrm{GHz}$, within $1 \%$ of the designed value of $15 \mathrm{GHz}$. At $14.95 \mathrm{GHz}$ the return loss was $22 \mathrm{~dB}$ indicating that there was no significant detrimental effect having the two switching diodes in series with the traveling wave channel.

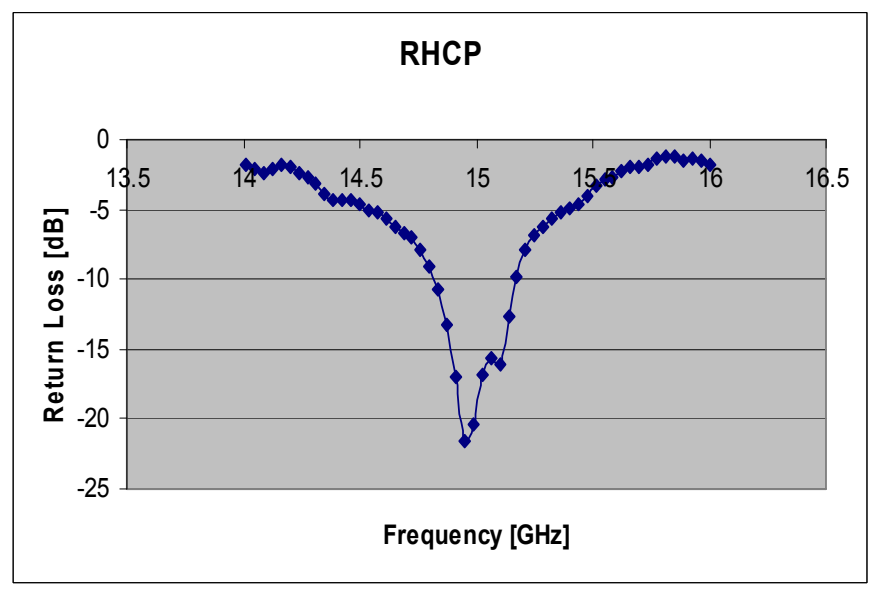

Fig.7. Typical measured return loss for one of the states of the antenna.

The primary reason for using an annular array of patches is to provide high quality circular polarization, in terms of a low axial ratio. A plot of the axial ratio as a function of frequency is shown in Fig. 8. It can be seen that at the optimum matching frequency of $14.95 \mathrm{GHz}$, the axial ratio is of the order of $0.8 \mathrm{~dB}$. This is a significantly better figure than is normally obtained with other forms of microstrip CP antenna [4]. The axial ratio bandwidth of the antenna is $3.4 \%$.

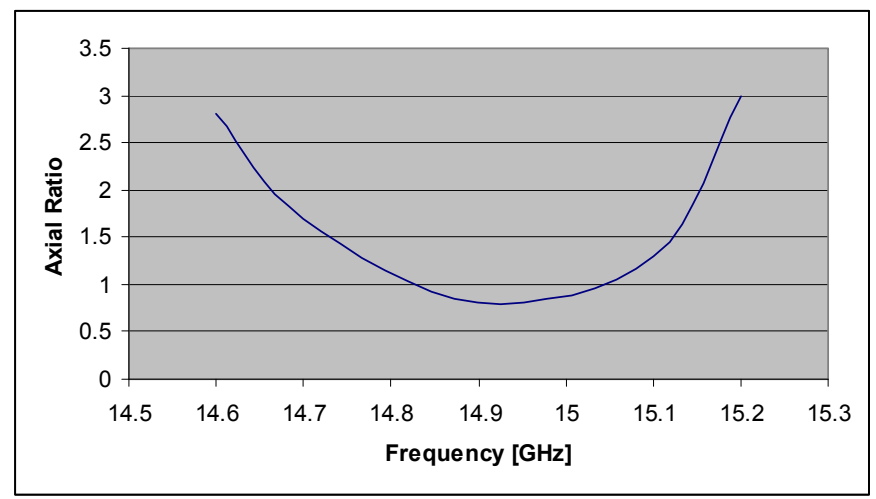

Fig. 8 Measured axial ratio

A typical radiation plot for the antenna is shown in Fig. 9. It can be seen that the antenna provides a well defined pattern, with the principal sidelobes $10 \mathrm{~dB}$ down on the boresight value. 


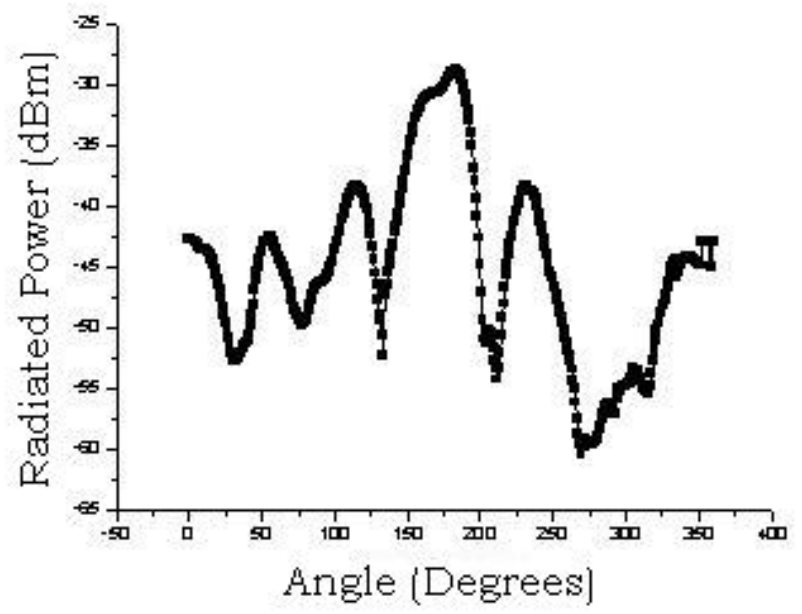

Fig. 9 Measured radiation characteristics at the optimum frequency of operation.

For this type of traveling wave antenna it is important, from the point of view of antenna efficiency, that minimum power is dissipated in the loads terminating the slotline channels.

To verify this, the through loss of the slotline channels was measured, and a typical result is shown in Fig. 10. In this figure the through loss is represented by $\left|\mathrm{S}_{21}\right|$. It can be seen that the loss is high over the frequency band of interest, indicating that the majority of the applied power is being coupled to the patches and radiated, although there will be some small dissipative losses in the material of the antenna.

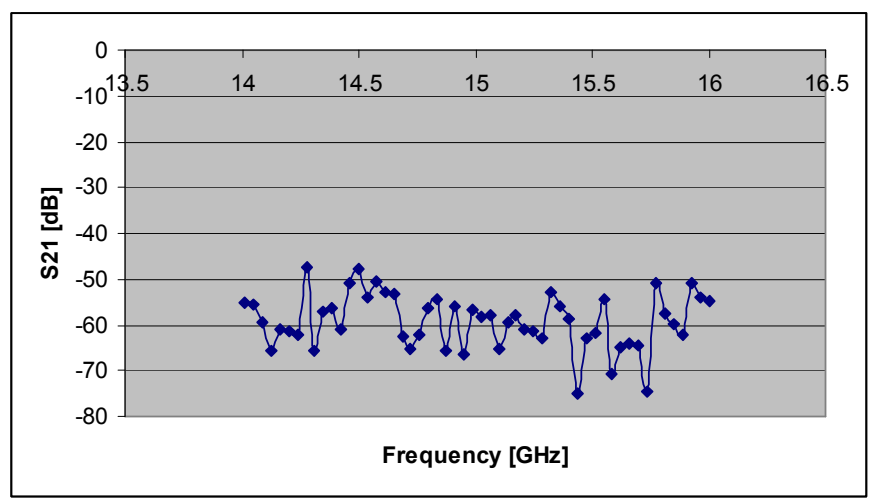

Fig. 10 Typical measured through loss of a slotline channel.

\section{DISCUSSION}

The measured performance of the antenna was close to the predicted performance, indicating that the design technique was fairly robust.

The antenna offers a number of beneficial features:

(i) The use of a multilayer structure, with mixed dielectric constant, proved useful in enhancing the radiation from the patches, whilst maintaining a small overall size for the antenna.

(ii) The antenna offers significant potential for further integration, by closely coupling the front-end circuitry of a transceiver in a layer beneath the feed network.

(iii) The design offers significant opportunities to increase the functionality of the antenna. Further slotline channels incorporating switching elements could be used to obtain linear polarization. Also, additional rings of patches could be used to broaden the bandwidth.

\section{CONCLUSIONS}

The concept of a new dual circular polarized active antenna, with the capability of switching between right and left hand circular polarization has been established through practical measurement. Opportunities have been identified to extend the design to a highly integrated smart antenna, having high functionality.

\section{REFERENCES}

[1] K.M. Lum; T. Tick; C. Free; H. Jantunen, "Design and Measurement Data for a Microwave Dual-CP Antenna Using a New Traveling-Wave Feed Concept", IEEE Trans. On Microwave Theory and Techniques, Vol. 54, no. 6, pp. 2880-2886, June 2006.

[2] K. M. Lum, C. Laohapensaeng, and C. E. Free, "A novel traveling-wave feed technique for circularly polarized planar antennas," IEEE Microwave Wireless Components Letters, vol. 15, no. 3, pp. 180-182, March 2005

[3] E. A. Mariani, C. P. Heinzman, J. P. Agrios, and S. B. Cohn, "Slot line characteristic", IEEE Trans. Microwave Theory Tech., vol. 17, no 12, pp. 1091-1096, December 1969.

[4] J. Huang, "A technique for an array to generate circular polarization with linearly polarized elements", IEEE Trans. Antennas and Propagation., vol. 34, no. 9, pp. 1113-1124, September 1986. 